\title{
Copula-Based Dynamic Conditional Correlation Multiplicative Error Processes
}

\author{
Taras Bodnar* \\ Nikolaus Hautsch**
}

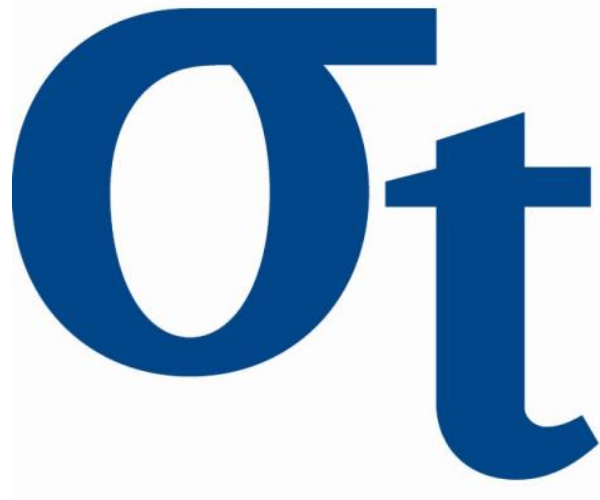

* European University Viadrina, Germany ** Humboldt-Universität zu Berlin, Germany
This research was supported by the Deutsche Forschungsgemeinschaft through the SFB 649 "Economic Risk".

http://sfb649.wiwi.hu-berlin.de ISSN 1860-5664

SFB 649, Humboldt-Universität zu Berlin Spandauer Straße 1, D-10178 Berlin
O

$\nabla$

(1)

ด

느

0 


\title{
Copula-Based Dynamic Conditional Correlation Multiplicative Error Processes *
}

\author{
Taras Bodnar ${ }^{\dagger} \quad$ Nikolaus Hautsch ${ }^{\ddagger}$
}

June 2012

\begin{abstract}
We introduce a copula-based dynamic model for multivariate processes of (non-negative) high-frequency trading variables revealing time-varying conditional variances and correlations. Modeling the variables' conditional mean processes using a multiplicative error model we map the resulting residuals into a Gaussian domain using a Gaussian copula. Based on high-frequency volatility, cumulative trading volumes, trade counts and market depth of various stocks traded at the NYSE, we show that the proposed copula-based transformation is supported by the data and allows disentangling (multivariate) dynamics in higher order moments. To capture the latter, we propose a DCC-GARCH specification. We suggest estimating the model by composite maximum likelihood which is sufficiently flexible to be applicable in high dimensions. Strong empirical evidence for time-varying conditional (co-)variances in trading processes supports the usefulness of the approach. Taking these higher-order dynamics explicitly into account significantly improves the goodness-of-fit of the multiplicative error model and allows capturing time-varying liquidity risks.
\end{abstract}

Keywords: multiplicative error model, trading processes, copula, DCC-GARCH, liquidity risk

JEL classification: C32, C58, C46

${ }^{*}$ This research is supported by the Deutsche Forschungsgemeinschaft via the Collaborative Research Center 649 "Economic Risk".

${ }^{\dagger}$ Department of Statistics, European University Viadrina. email: bodnar@europa-uni.de. Address: PO Box 1786, 15207 Frankfurt (Oder), Germany.

${ }^{\ddagger}$ Institute for Statistics and Econometrics and Center for Applied Statistics and Economics (CASE), HumboldtUniversität zu Berlin as well as Center for Financial Studies (CFS), Frankfurt. Email: nikolaus.hautsch@wiwi.huberlin.de. Address: Spandauer Str. 1, D-10178 Berlin, Germany. 


\section{Introduction}

The modeling of intraday trading processes has become a major area in financial econometrics. This is particularly triggered by technological progress on financial markets, changing institutional structures in the trading landscape and a growing importance of intraday trading. The availability of financial data on the lowest possible aggregation level opens up the possibility to gain a deeper understanding of financial trading processes and to successfully manage trading risks, trading costs and intraday price risks.

This paper contributes to the literature on multivariate models for trading processes. We propose a model capturing trading dynamics not only in first conditional moments but also in conditional (co-)variances. The latter reflect time-varying uncertainty inherent in intraday trading processes as well as dynamic correlation structures between key trading variables. The major idea is to map innovations in (non-negative) dynamic trading processes into a Gaussian domain using a Gaussian copula. The innovations stem from a vector multiplicative error model (VMEM) as proposed by Manganelli (2005) and Cipollini, Engle, and Gallo (2007). The copula-based Gaussian transformation of observations allows identifying non-linear dependencies between trading variables and yields a natural separation of (multivariate) dynamics in first and second conditional moments. The latter are conveniently captured using dynamic conditional correlation (DCC) models as proposed by Engle (2002a). The proposed approach is sufficiently flexible to be applicable in high dimensions and can be extended in various directions.

Multiplicative error models (MEMs) - labeled according to Engle (2002b) - are workhorses for the modeling of dynamic processes of non-negative random variables, such as trading volumes, volatilities, trading intensities or market depth. The principle of decomposing a process into the product of its conditional mean and a positive-valued error term is well known in the literature and builds the backbone of the autoregressive conditional heteroscedasticity (ARCH) model by Engle (1982) and the stochastic volatility (SV) model introduced by Taylor (1982). In high-frequency econometrics, it is put forward by Engle and Russell (1998) to model the dynamics of trade-to-trade durations and is referred to as autoregressive conditional duration (ACD) model. $^{1}$

A difficulty in the modeling of non-negative random variables is that typical distributions, such as the exponential distribution or generalizations thereof, imply a direct relationship between all moments. Accordingly, a MEM process implies that higher order (conditional) mo-

\footnotetext{
${ }^{1}$ See, e.g., Hautsch (2012) for an overview.
} 
ments follow the same dynamics which, however, is not necessarily supported by the data. To address this problem, Ghysels, Gouriéroux, and Jasiak (1998) propose a two-factor model allowing for separate dynamics of the conditional mean and the conditional variance. Their principle is to rewrite an exponential model with gamma heterogeneity in terms of two Gaussian factors following a bi-variate dynamic process. Though this model accounts for features in trading variables which are not captured by a basic MEM, it imposes (partly restrictive) distributional assumptions and is hard to estimate. In a multivariate setting, the situation is even more complicate as not only conditional variance dynamics but also time-varying correlation structures have to be taken into account. However, finding a sufficiently flexible multivariate distribution defined on positive support is a difficult task. As discussed by Cipollini, Engle, and Gallo (2007), a possible candidate is a multivariate gamma distribution which however imposes severe restrictions on the contemporaneous correlations between the variables.

This paper's contribution is to capture higher-order dependence structures using a copulabased decomposition of dynamics. Capturing conditional mean dynamics using a VMEM specification, the resulting residuals serve as serially uncorrelated innovations whose multivariate distribution is modeled using a copula. Employing a Gaussian copula has two major advantages: First, the copula allows to straightforwardly link the individual marginal distributions to an appropriate joint distribution. Moreover, the imposed normality enables naturally disentangling first and second conditional moments. Furthermore, the mapping into a Gaussian domain allows identifying non-linear (cross-)dependencies in trading processes which are not identifiable using a basic (linear) VMEM. The dynamics in resulting transformed innovations are naturally captured using (V)ARMA-GARCH and DCC-type specifications. This makes the model quite flexible and applicable in high dimensions. Accordingly, we suggest a composite maximum likelihood estimation procedure which is also feasible for high-dimensional processes.

We apply the model to 5-min squared mid-quote returns, cumulative trading volumes, trade counts as well as market depth of different stocks traded at the New York Stock Exchange (NYSE). We show that the Gaussian copula and thus the normality-induced separation between first and second conditional moments is well supported by the data. It turns out that VMEM innovations still reveal substantial dependencies in higher moments which are only identifiable after the application of the Gaussian copula. It turns out that the explicit consideration of these dependencies lead to a significantly better fit in terms of information criteria.

Our study shows that trading variables are subject to time-varying conditional variances reflecting liquidity and volatility risk. The processes are quite persistent and reveal positive cross- 
dependencies. Hence, uncertainty in volatility as well as liquidity demand and supply tends to spill over from one variable to another. Moreover, we show that conditional correlations between liquidity and volatility variables substantially vary over time. These insights are interesting from a microstructure and trading perspective as they allow identifying periods where connections between liquidity demand, liquidity supply and volatility are particularly high or low, respectively. Residual diagnostics show that the proposed approach explains the multivariate dynamics in trading processes clearly better than a basic (linear) VMEM specification.

The proposed copula-based dynamic conditional correlation MEM complements the existing literature on multiplicative error processes and the modeling of intraday trading. Various aspects which have been addressed in extant literature can be included in our approach. For instance, latent factor approaches in the spirit of Bauwens and Veredas (2004) and Hautsch (2008), component MEMS, as proposed by Brownlees, Cipollini, and Gallo (2010) or Brownlees and Vannucci (2010), long memory dynamics, as put forward by Jasiak (1998) and Karanasos (2004), or regime-switching MEMs as in Zhang, Russell, and Tsay (2001) or Meitz and Teräsvirta (2006) could easily be included in the basic (V)MEM specification. Likewise, the included DCC-GARCH component could be further extended by recent advances in the literature on multivariate GARCH models (see, e.g., Bauwens, Laurent, and Rombouts (2006)). Finally, our approach contributes to the empirical literature on dynamic copula models, see, e.g., Patton (2001) and Patton (2006), or on copula-based multivariate GARCH processes as suggested by Jondeau and Rockinger (2006), Lee and Long (2009) and Liu and Luger (2009). A study related to ours is Cipollini, Engle, and Gallo (2007) who also suggest copulas in a (V)MEM setup but proceed differently.

The remainder of the paper is organized as follows. Section 2 briefly reviews the basic vector multiplicative error model. Section 3 introduces the new copula-based approach. In Section 4 , we provide an empirical application to the modeling of high-frequency trading processes. Finally, Section 5 concludes.

\section{The Basic Multiplicative Error Model}

Let $\left\{X_{t}\right\}, t=1, \ldots, T$, denote a non-negative valued random process and let $\mathcal{F}_{t}$ define the information set up to time $t$. The basic univariate multiplicative error model (MEM), as introduced 
by Engle (2002b), is given by

$$
\begin{aligned}
X_{t} & =\mu_{t} \varepsilon_{t}, \\
\varepsilon_{t} \mid \mathcal{F}_{t-1} & \sim \text { i.i.d. } D\left(1, \sigma^{2}\right),
\end{aligned}
$$

where $\left\{\mu_{t}\right\}$ is a non-negative random predictable process with respect to $\mathcal{F}_{t-1}$ and represents the conditional mean of $X_{t}$. The class of MEMs is quite general and nests various special cases. For instance, Bollerslev's (1986) generalized autoregressive conditional heteroscedasticity model is obtained by letting $X_{t}$ be the squared de-meaned log return $Y_{t}$ with $\mu_{t}$ being its conditional variance, i.e., $Y_{t}^{2}=\mu_{t} \varepsilon_{t}^{2}$ or, alternatively, $Y_{t}=\sqrt{\mu_{t}} \varepsilon_{t}$ with $\varepsilon_{t} \mid \mathcal{F}_{t-1} \sim$ i.i.d. $(0,1)$. Another important special case of the univariate MEM is the autoregressive conditional duration (ACD) model proposed by Engle and Russell (1998), where $X_{t}$ corresponds to a financial duration.

The conditional mean $\mu_{t}$ can be parameterized in various ways. In its most simple form, it is specified as

$$
\mu_{t}=\omega+\alpha_{1} X_{t-1}+\beta_{1} \mu_{t-1}
$$

corresponding to an $\operatorname{ARMA}(1,1)$ process for $X_{t}$ with (martingale difference) error term $X_{t}-\mu_{t}$. Exploiting the analogy to a GARCH process, (univariate) MEMs can be extended in various ways. For a recent overview, see Hautsch (2012).

Multivariately extending the MEM yields the so-called vector multiplicative error model (VMEM) given by

$$
\mathbf{X}_{t}=\boldsymbol{\mu}_{t} \odot \varepsilon_{t}
$$

where $\mathbf{X}_{t}:=\left(\mathbf{X}_{1, t}, \ldots, \mathbf{X}_{K, t}\right)^{\prime}, \boldsymbol{\mu}_{t}:=\mathrm{E}\left[\mathbf{X}_{t} \mid \mathcal{F}_{t-1}\right]=\left(\boldsymbol{\mu}_{1, t}, \ldots, \boldsymbol{\mu}_{K, t}\right)^{\prime}$ is a $K \times 1$ vector, ' $\odot$ ' denotes the Hadamard product (element-wise multiplication) and $\varepsilon_{t}$ is a $K$-dimensional vector of mutually and serially i.i.d. innovation processes, where the $j$-th element is given by

$$
\varepsilon_{j, t} \mid \mathcal{F}_{t-1} \sim D^{j}\left(1, \sigma_{j}^{2}\right)
$$

Manganelli (2005) suggests specifying $\boldsymbol{\mu}_{t}$ as

$$
\boldsymbol{\mu}_{t}=\boldsymbol{\omega}+\mathbf{A}_{0} \mathbf{X}_{t}+\sum_{p=1}^{P} \mathbf{A}_{p} \mathbf{X}_{t-p}+\sum_{q=1}^{Q} \mathbf{B}_{q} \boldsymbol{\mu}_{t-q},
$$

where $\boldsymbol{\omega}$ is a $K$-dimensional vector and $\mathbf{A}_{0}, \mathbf{A}_{p}, \mathbf{B}_{q}$ are $K \times K$ parameter matrices. The matrix $\mathbf{A}_{0}$ captures the contemporaneous dependence of the elements of $\mathbf{X}_{t}$ and is specified as a matrix 
where only the upper triangular elements are non-zero. Consequently, $X_{i, t}$ is predetermined for all variables $X_{j, t}, j<i$, and is conditionally i.i.d. given $\left\{X_{j, t}, \mathcal{F}_{t-1}\right\}, j<i$.

The disadvantage of this structure is the requirement of imposing an explicit ordering of the variables in $\mathbf{X}_{t}$ induced by the triangular structure. The latter is not easy to justify in most applications. Accordingly, mutual dependencies are likely to be misspecified. This problem becomes even more severe when the dimension of the underlying process is high. An alternative way to capture contemporaneous relationships is to allow for mutual correlations between the innovation terms $\varepsilon_{i, t}$. Then, the innovation terms follow a density function which is defined over non-negative $K$-dimensional support $[0,+\infty)^{K}$ with unit mean $\iota$ and covariance matrix $\boldsymbol{\Sigma}$, i.e.,

$$
\varepsilon_{t} \mid \mathcal{F}_{t-1} \sim \text { i.i.d. } \mathcal{D}(\iota, \Sigma)
$$

implying

$$
\begin{aligned}
\mathrm{E}\left[\mathbf{X}_{t} \mid \mathcal{F}_{t-1}\right] & =\boldsymbol{\mu}_{t}, \\
\mathrm{~V}\left[\mathbf{X}_{t} \mid \mathcal{F}_{t-1}\right] & =\boldsymbol{\mu}_{t} \boldsymbol{\mu}_{t}^{\prime} \odot \boldsymbol{\Sigma} .
\end{aligned}
$$

However, finding an appropriate distribution $\mathcal{D}$ is a difficult task. Typical distributions for positive-valued random variables impose direct relationships between all moments. To break up this dependence, Ghysels, Gouriéroux, and Jasiak (2004) propose a two-factor model which allows estimating separate dynamics for the conditional variance of durations ('duration volatilities') leading to the so-called Stochastic Volatility Duration model. In a multivariate context, the separation between first and second (conditional) moments is even more challenging. As discussed by Cipollini, Engle, and Gallo (2007), a possible candidate for $\mathcal{D}$ is a multivariate gamma distribution which, however, imposes severe restrictions on the contemporaneous correlations between the errors $\varepsilon_{j, t}$.

Below we propose a copula-based approach which (i) allows to separately model first and second moments and (ii) is tractable and feasible even in high dimensions.

\section{Copula-Based Dynamic Conditional Correlation Multiplicative Er- ror Processes}

\subsection{The Model}

The major idea is to find an appropriate transformation of $\varepsilon_{t}$ which allows disentangling first and second moments. Such a transformation step is in the spirit of Ghysels, Gouriéroux, and Jasiak 
(2004) who propose rewriting an exponential model with gamma heterogeneity in terms a twofactor formulation based on Gaussian factors. The latter are individually modeled using standard time series approaches. However, the disadvantage of the so-called Stochastic Volatility Duration model is that it is computationally expensive and difficult to be generalized to a multivariate setting.

As an alternative we suggest a transformation step which is computationally tractable (and thus feasible for large data sets) and also easily applicable in high dimensions. The conditional mean of $\mathbf{X}_{t}$ is specified according to a simplified version of (4),

$$
\begin{aligned}
\mathbf{X}_{t} & =\boldsymbol{\mu}_{t} \odot \varepsilon_{t} \\
\boldsymbol{\mu}_{t} & =\boldsymbol{\omega}+\sum_{p=1}^{P} \mathbf{A}_{p} \mathbf{X}_{t-p}+\sum_{q=1}^{Q} \mathbf{B}_{q} \boldsymbol{\mu}_{t-q},
\end{aligned}
$$

with the elements of $\varepsilon_{t}$ being uncorrelated (but not independent). Note that (5) excludes contemporaneous dependencies between the elements of $\mathbf{X}_{t}$ (in contrast to (4)), as they are exclusively captured by mutual dependencies in $\varepsilon_{j, t}$.

The major principle is to transform $\varepsilon_{t}$ to the vector $\varepsilon_{t}^{*}$ using a Gaussian copula, i.e.,

$$
\varepsilon_{t}^{*}:=\left(\Phi^{-1}\left(F_{1}\left(\varepsilon_{1, t}\right)\right), \ldots, \Phi^{-1}\left(F_{K}\left(\varepsilon_{K, t}\right)\right)\right)^{\prime},
$$

where $\Phi($.$) denotes the c.d.f. of the univariate standard normal distribution and F_{j}($.$) denotes$ the marginal cumulative distribution function associated with $D^{j}$. The assumption of the Gaussian copula implies that the transformed residuals $\varepsilon_{t}^{*}$ are by construction conditionally normally distributed,

$$
\varepsilon_{t}^{*} \mid \mathcal{F}_{t-1} \sim \mathcal{N}\left(\boldsymbol{\mu}_{t}^{*}, \mathbf{D}_{t} \mathbf{R}_{t} \mathbf{D}_{t}\right)
$$

with $\boldsymbol{\mu}_{t}^{*}$ denoting the conditional mean, $\mathbf{R}_{t}$ denoting the conditional correlation matrix and $\mathbf{D}_{t}$ are $K \times K$ diagonal matrices with the conditional volatilities $h_{j, t}^{1 / 2}, j=1, \ldots K$, as diagonal elements.

The transformation underlying the Gaussian copula can be interpreted as a mapping of an observation $\mathbf{X}_{t}$ from the support of $D^{1} \times D^{2} \times \ldots \times D^{K}$ on the support of the marginal c.d.f. of a $K$-dimensional normal distribution with the respective quantiles being equal. Since the transformation from $\varepsilon_{t}$ to $\varepsilon_{t}^{*}$ is non-linear (though monotone), the series $\left\{\varepsilon_{t}^{*}\right\}$ as well as $\left\{\varepsilon_{t}^{* 2}\right\}$ might be autocorrelated while the $\left\{\varepsilon_{t}\right\}$ themselves are uncorrelated. Indeed, the existence of dynamics in $\varepsilon_{t}^{*}$ and $\varepsilon_{t}^{* 2}$ indicate the presence of higher-order dynamics in $\varepsilon_{t}$ which are not captured by the plain MEM specification. Likewise, non-zero (conditional) correlations between $\varepsilon_{j, t}^{*}$, 
$j=1, \ldots, K$, reflect the presence of (time-varying) mutual dependencies between MEM errors. Clearly, the adequacy of the Gaussian copula has to be empirically validated by testing the normality of $\varepsilon_{t}^{*}$. Our empirical findings below show that this assumption is well supported by typical high-frequency time series.

A mapping into the Gaussian domain has the advantage of fully describing all dependencies in terms of the first two conditional moments of the transformed error process. As a result, timevariations in higher-order dependencies can be conveniently captured by dynamic conditional variance and correlation processes bringing well-known time series approaches into play. Indeed, such a modeling strategy is not feasible if it is directly applied to $\varepsilon_{t}$ as the amount of information presented in $\left\{\varepsilon_{t}\right\}$ is not revealed without appropriately transforming $\left\{\varepsilon_{t}\right\}$.

To capture possible dynamics in the first two conditional moments of $\varepsilon_{t}^{*}$, we propose an VAR-(M)GARCH parameterization given by

$$
\begin{aligned}
& \boldsymbol{\varepsilon}_{t}^{*}=\boldsymbol{\mu}_{t}^{*}+\boldsymbol{\eta}_{t}=: \sum_{j=1}^{Q^{*}} \mathbf{C}_{j} \varepsilon_{t-j}^{*}+\boldsymbol{\eta}_{t}, \\
& \boldsymbol{\eta}_{t}=\sqrt{\mathbf{h}_{t}} \odot \boldsymbol{\nu}_{t}, \\
& \mathbf{h}_{t}=\boldsymbol{\omega}_{h}+\sum_{j=1}^{P^{h}} \mathbf{A}_{j}^{h}\left(\boldsymbol{\eta}_{t-j} \odot \boldsymbol{\eta}_{t-j}\right)+\sum_{j=1}^{Q^{h}} \mathbf{B}_{j}^{h} \mathbf{h}_{t-j},
\end{aligned}
$$

where $\mathbf{C}_{j}, \mathbf{A}_{j}^{h}$ and $\mathbf{B}_{j}^{h}$ are $K \times K$ parameter matrices. Correspondingly, $\mathbf{h}_{t}$ is a $K$-vector of conditional variances $h_{j, t}, j=1, \ldots, K$, of $\boldsymbol{\eta}_{t}$ and $\boldsymbol{\nu}_{t}$ is a $K$-vector with $\boldsymbol{\nu}_{t} \mid \mathcal{F}_{t-1} \sim \mathcal{N}\left(\mathbf{0}, \mathbf{R}_{t}\right)$.

Obviously, $h_{i, t}$ is not the conditional variance of $\varepsilon_{i, t}$ but the conditional variance of its projection into a Gaussian domain. Nevertheless, it can be seen as an approximation of the conditional variance of $\varepsilon_{i, t}$ reflecting normalized fluctuations around the conditional mean. As the underlying transformation is monotonous and increasing, a higher (lower) volatility in the process $\left\{\varepsilon_{t}^{*}\right\}$ also indicates a higher (lower) volatility in the process $\left\{\varepsilon_{t}\right\}$.

The conditional correlation matrix $\mathbf{R}_{t}$ is modeled according to Engle's (2002a) Dynamic Conditional Correlation (DCC) model and is given by

$$
\begin{aligned}
& \mathbf{R}_{t}=\mathbf{Q}_{t}^{*-1} \mathbf{Q}_{t} \mathbf{Q}_{t}^{*-1}, \\
& \mathbf{Q}_{t}=\left(1-\sum_{j=1}^{P^{R}} \gamma_{j}-\sum_{j=1}^{Q^{R}} \delta_{j}\right) \overline{\mathbf{Q}}+\sum_{j=1}^{P^{R}} \gamma_{j} \boldsymbol{\nu}_{t-j} \boldsymbol{\nu}_{t-j}^{\prime}+\sum_{j=1}^{Q^{R}} \delta_{j} \mathbf{Q}_{t-j},
\end{aligned}
$$

where $\overline{\mathbf{Q}}$ is the unconditional covariance matrix of $\boldsymbol{\nu}_{t}$.

The process $\left\{\varepsilon_{t}^{*}\right\}$ is weakly stationary if (i) all eigenvalues of the matrices $\sum_{j=1}^{Q^{*}} \mathbf{C}_{j}$ and $\sum_{j=1}^{P^{h}} \mathbf{A}_{j}^{h}+\sum_{j=1}^{Q^{h}} \mathbf{B}_{j}^{h}$ have a modulus smaller than one, and (ii) $\sum_{j=1}^{P^{R}} \gamma_{j}+\sum_{j=1}^{Q^{R}} \delta_{j}<1$. 
These conditions ensure the weak stationarity of $\left\{\boldsymbol{\nu}_{t}\right\}$ and $\left\{\varepsilon_{t}^{*}\right\}$. Weak stationarity of $\left\{\varepsilon_{t}\right\}$ is guaranteed by the existence of the first two moments of $\left\{\varepsilon_{t}\right\}$. Then, the process $\left\{\varepsilon_{t}\right\}$ is obtained from $\left\{\varepsilon_{t}^{*}\right\}$ by applying a time-invariant transformation, guaranteeing the weak stationarity of the process $\left\{\varepsilon_{t}\right\}$. Finally, the weak stationarity of $\left\{\mathbf{X}_{t}\right\}$ is satisfied by the weak stationarity of $\left\{\boldsymbol{\mu}_{t}\right\}$ requiring all eigenvalues of the matrix $\sum_{p=1}^{P} \mathbf{A}_{p}+\sum_{q=1}^{Q} \mathbf{B}_{q}$ having modulus smaller than one.

Then, the conditional moments of $\mathbf{X}_{t}$ are given by

$$
\begin{aligned}
\mathrm{E}\left[\mathbf{X}_{t} \mid \mathcal{F}_{t-1}\right] & =\boldsymbol{\mu}_{t}, \\
\mathrm{~V}\left[X_{j, t} \mid \mathcal{F}_{t-1}\right] & =\mu_{j, t}^{2} \sigma_{j}^{2}, \\
\operatorname{Cov}\left(X_{i, t}, X_{j, t} \mid \mathcal{F}_{t-1}\right) & =\mu_{i, t} \mu_{j, t} \operatorname{Cov}\left(\varepsilon_{i, t}, \varepsilon_{j, t} \mid \mathcal{F}_{t-1}\right) \\
& =\mu_{i, t} \mu_{j, t} \operatorname{Cov}\left(F_{i}^{-1}\left(\Phi\left(\varepsilon_{i, t}^{*}\right)\right), F_{j}^{-1}\left(\Phi\left(\varepsilon_{j, t}^{*}\right)\right) \mid \mathcal{F}_{t-1}\right)
\end{aligned}
$$

Note that the last integral cannot be evaluated analytically but can be easily computed numerically.

\subsection{Statistical Inference}

The joint density of the $\varepsilon_{t}$ 's is given by

$$
f_{\boldsymbol{\varepsilon}_{t} \mid \mathcal{F}_{t-1}}\left(\varepsilon_{1 ; t}, \ldots, \varepsilon_{K ; t}\right)=\phi_{K}\left(C(L) \varepsilon_{t}^{*}, \mathbf{D}_{t} \mathbf{R}_{t} \mathbf{D}_{t} ; \varepsilon_{t}^{*}\right) \prod_{j=1}^{K} \frac{f_{j}\left(\varepsilon_{j, t}\right)}{\phi\left(\Phi^{-1}\left(F_{j}\left(\varepsilon_{j, t}\right)\right)\right)}
$$

where $\phi_{K}(\boldsymbol{\mu}, \boldsymbol{\Sigma} ;$.$) is the probability density function of the K$-dimensional multivariate normal distribution with mean vector $\boldsymbol{\mu}$ and covariance matrix $\boldsymbol{\Sigma}, \phi($.$) denotes the density function of$ the (univariate) standard normal distribution, $f_{j}($.$) is the marginal density function of \varepsilon_{j, t}$ and $C(L):=\sum_{p=1}^{Q^{*}} \mathbf{C}_{p} L^{p}$ with $L$ representing the lag operator. From the transformations $X_{j, t}=$ $\mu_{j, t} \varepsilon_{j, t}$ with the Jacobian $\prod_{j=1}^{k} \mu_{j, t}^{-1}$ we can deduce the joint conditional density of $\mathbf{X}_{t}$ given $\mathcal{F}_{t-1}$,

$$
f_{\mathbf{X}_{t} \mid \mathcal{F}_{t-1}}\left(X_{1 ; t}, \ldots, X_{K ; t}\right)=\phi_{K}\left(C(L) \mathbf{q}_{t}, \mathbf{D}_{t} \mathbf{R}_{t} \mathbf{D}_{t} ; \mathbf{q}_{t}\right) \prod_{j=1}^{K} \frac{\mu_{j, t}^{-1} f_{j}\left(\frac{X_{j, t}}{\mu_{j, t}}\right)}{\phi\left(\Phi^{-1}\left(F_{j}\left(\frac{X_{j, t}}{\mu_{j, t}}\right)\right)\right.}
$$

where $\mathbf{q}_{t}:=\left[\Phi^{-1}\left(F_{1}\left(\frac{X_{1, t}}{\mu_{1, t}}\right)\right), \ldots, \Phi^{-1}\left(F_{K}\left(\frac{X_{K, t}}{\mu_{K, t}}\right)\right)\right]^{\prime}$.

Let $\boldsymbol{\theta}$ denote the vector of parameters. Given the data matrix $\mathbf{W}$, the $\log$ likelihood function 
is calculated employing (14) and is given by

$$
\begin{aligned}
l(\boldsymbol{\theta} ; \mathbf{W}) & =\sum_{t=1}^{T} \sum_{j=1}^{K} \ln \left(f_{j}\left(\frac{X_{j, t}}{\mu_{j, t}}\right)\right)-\sum_{t=1}^{T} \sum_{j=1}^{K} \ln \left(\mu_{j, t}\right) \\
& +\sum_{t=1}^{T} \ln \left(\phi_{K}\left(C(L) \mathbf{q}_{t}, \mathbf{D}_{t} \mathbf{R}_{t} \mathbf{D}_{t} ; \mathbf{q}_{t}\right)\right)-\sum_{t=1}^{T} \sum_{j=1}^{K} \ln \left[\phi\left\{\Phi^{-1}\left(F_{j}\left(\frac{X_{j, t}}{\mu_{j, t}}\right)\right)\right\}\right] \\
& =l_{0}+l_{1},
\end{aligned}
$$

where

$$
\begin{aligned}
& l_{0}:=\sum_{t=1}^{T} \sum_{j=1}^{K} \ln \left(f_{j}\left(\frac{X_{j, t}}{\mu_{j, t}}\right)\right)-\sum_{t=1}^{T} \sum_{j=1}^{K} \ln \left(\mu_{j, t}\right), \\
& l_{1}:=\sum_{t=1}^{T} \ln \left(\phi_{K}\left(C(L) \mathbf{q}_{t}, \mathbf{D}_{t} \mathbf{R}_{t} \mathbf{D}_{t} ; \mathbf{q}_{t}\right)\right)-\sum_{t=1}^{T} \sum_{j=1}^{K} \ln \left[\phi\left\{\Phi^{-1}\left(F_{j}\left(\frac{X_{j, t}}{\mu_{j, t}}\right)\right)\right\}\right] .
\end{aligned}
$$

The structure of the likelihood function (15) motivates the application of a multi-step composite likelihood procedure. Let the symbol vec denote the vec-operator and $\boldsymbol{\theta}:=\left(\boldsymbol{\theta}_{0}^{\prime}, \boldsymbol{\theta}_{1}^{\prime}\right)^{\prime}$ with

$$
\begin{aligned}
\boldsymbol{\theta}_{0}:= & \left(\boldsymbol{\omega}^{\prime}, \operatorname{vec}\left(\mathbf{A}_{1}\right)^{\prime}, \ldots, \operatorname{vec}\left(\mathbf{A}_{P}\right)^{\prime}, \operatorname{vec}\left(\mathbf{B}_{1}\right)^{\prime}, \ldots, \operatorname{vec}\left(\mathbf{B}_{Q}\right)^{\prime}\right)^{\prime} \\
\boldsymbol{\theta}_{1}:= & \left(\operatorname{vec}\left(\mathbf{C}_{1}\right)^{\prime}, \ldots, \operatorname{vec}\left(\mathbf{C}_{Q^{*}}\right)^{\prime}, \boldsymbol{\omega}_{h}^{\prime}, \operatorname{vec}\left(\mathbf{A}_{1}^{h}\right)^{\prime}, \ldots, \operatorname{vec}\left(\mathbf{A}_{P^{h}}^{h}\right)^{\prime}, \operatorname{vec}\left(\mathbf{B}_{1}^{h}\right)^{\prime}, \ldots, \operatorname{vec}\left(\mathbf{B}_{Q^{h}}^{h}\right)^{\prime},\right. \\
& \left.\gamma_{1}, \ldots, \gamma_{P^{R}}, \delta_{1}, \ldots, \delta_{Q^{R}}\right)^{\prime}
\end{aligned}
$$

being the parameters of the plain VECM and VAR-(M)GARCH part of the model, respectively. Accordingly, the likelihood function of the extended VECM process consists of $K+1$ (conditional) components. The first $K$ components are specified by the conditional distribution of each component of $\mathbf{X}_{t}$ given $\mathcal{F}_{t-1}$ and are fully determined by the parameter vector $\boldsymbol{\theta}_{0}$ and the distribution functions $F_{j}(),. j=1, \ldots, K$. The $(K+1)$-th component is given by the conditional distribution of the VAR-(M)GARCH part, i.e., by the conditional distribution of $\mathbf{q}_{t}$, which is a conditional normal distribution with conditional mean vector $\boldsymbol{\mu}^{*}=C(L) \mathbf{q}_{t}$ and conditional covariance matrix $\mathbf{D}_{t} \mathbf{R}_{t} \mathbf{D}_{t}$. Using this presentation we adopt a two stage procedure. To compute $l_{0}$, we choose $f_{j}(),. j=1, \ldots, K$ as the densities of (conditional) exponential distributions. Accordingly, we have

$$
l_{0}=\sum_{t=1}^{T} \sum_{j=1}^{K} \frac{X_{j, t}}{\mu_{j, t}}-\sum_{t=1}^{T} \sum_{j=1}^{K} \log \left(\mu_{j, t}\right)
$$

ignoring any constants. Maximizing $l_{0}$ yields estimates of $\boldsymbol{\theta}_{0}$. Exploiting the quasi-likelihood property of the exponential distribution (see, e.g., Engle (2000)), $\boldsymbol{\theta}_{0}$ and consequently first-stage 
MEM residuals are consistently estimated. In the second stage, $\boldsymbol{\theta}_{1}$ is estimated using MEM residuals. The unknown distribution functions $F_{j}($.$) of \varepsilon_{j, t}$ are replaced by the rescaled univariate

marginal empirical distributions $\widehat{\mathbb{F}}_{j}=\frac{T}{T+1} \hat{F}_{j}$, where $\hat{F}_{j}$ are the corresponding empirical distribution functions of $\hat{\varepsilon}_{j, t}$. Then, the resulting residuals $\hat{\varepsilon}_{t}^{*}=\left(\Phi^{-1}\left(\hat{F}_{1}\left(\varepsilon_{1, t}\right)\right), \ldots, \Phi^{-1}\left(\hat{F}_{k}\left(\varepsilon_{k, t}\right)\right)\right)^{\prime}$ are used to estimate $\boldsymbol{\theta}_{1}$. Consequently,

$$
l_{1}=\sum_{t=1}^{T} \ln \left(\phi_{K}\left(C(L) \hat{\varepsilon}_{t}^{*}, \mathbf{D}_{t} \mathbf{R}_{t} \mathbf{D}_{t} ; \hat{\varepsilon}_{t}^{*}\right)\right)
$$

is maximized over $\boldsymbol{\theta}_{1}$. Under some regularity conditions (see e.g. White (1996) and Engle and Sheppard (2001)), it can be shown that the suggested two-stage estimator is consistent and asymptotically normally distributed. For more details on composite likelihood approaches, see, e.g., Lindsay (1988), Joe (1997), Joe (2005) or Ng, Joe, Karlis, and Liu (2011).

\section{Modeling High-Frequency Volatility and Liquidity}

\subsection{Data}

We apply the model to five stocks traded at the New York Stock Exchange (NYSE) in 2006. To provide cross-sectional evidence across different liquidity classes, we choose the tickers AIG, Citigroup, Boeing, ASH and AMR. AIG and Citigroup belong to the biggest financial and insurance companies in the U.S., Boeing is one of the biggest multinational aerospace and defense companies, ASH represents Ashland Inc., a chemical and petroleum company while AMR Corporation is an aviation and airline holding company. We apply the model to equi-distant data and focus on 5-min aggregates. This allows analyzing a sufficiently long period (one year) without facing too much computational burden. As the variables of interest, we choose a volatility proxy, computed as the squared residual of an ARMA(1,1) regression of 5-min log mid-quote returns. Moreover, we use the cumulated trading volume, the number of trades as well as the time-weighted average depth on the ask and bid level. All variables are seasonally adjusted by standardizing them by a cubic spline $s_{j, t}, j=1, \ldots, K$, based on 30-min nodes covering the trading day from 9:30 to 16:00. Table 1 in the Appendix gives summary statistics of the underlying 5-min returns, scaled to an annual level, cumulative trading volumes, number of trades and average first-level depth (in number of round lots). We observe a high variability of returns which is becoming even higher in case of less liquid assets. For instance, if the 5-min returns would be scaled to an annual level, we would observe standard deviations of around 20. Substantial variations are also observed for cumulative trading volumes which, however, are obviously 
driven by underlying lot sizes. In terms of the number of trades, Citigroup and AIG are most intensively traded with on average approximately 45 trades per 5 minutes. Conversely, Boeing and AMR represent less liquid assets with on average approximately 34 trades per interval while ASH reveals the lowest liquidity trading on average 20 times per 5 minutes. As reflected by the Ljung-Box statistics, all series are strongly autocorrelated suggesting a dynamic model.

\subsection{Estimation Results}

We apply the model to the stock characteristics $\mathbf{X}_{t}$ with elements $X_{1, t}$ (volatility proxy, computed as the squared residual of an $\operatorname{ARMA}(1,1)$ regression of 5-min log mid-quote returns), $X_{2, t}$ (cumulative trading volumes), $X_{3, t}$ (number of trades), $X_{4, t}$ (average first-level depth) for $t=1, \ldots, T$. The estimates of the underlying VMEM specification are given in Table 3 . The lag orders are chosen using the Bayes Information Criterion (BIC) suggesting a $\operatorname{VMEM}(3,1)$ specification yielding the best fit. All processes are dominantly driven by their own histories implying the diagonal elements of $\mathbf{A}_{1}$ being largest and most significant. Nevertheless, also significant evidence for dynamic spill-overs between the individual variables is shown. We observe positive intertemporal dependencies between volatility and liquidity demand, i.e., high volatilities induce high trading volumes and trading intensities and vice versa. The same is true for the relationship between cumulative trading volumes and the number of trades. This is not surprising as there is an inherent link between cumulated volumes and the number of trades per time. Conversely, liquidity supply - as reflected by the pending depth on the first level - is negatively linked to volatility. Hence, in periods of high volatility, liquidity suppliers tend to reduce order aggressiveness and thus post less depth on the first level. This is in strong accordance with predictions from market microstructure theory, see, e.g., Glosten (1994). In contrast, there is a positive intertemporal relationship between liquidity supply and liquidity demand: A high liquidity demand - reflected by high trading volumes - increases execution probabilities of limit orders and thus rises liquidity suppliers' willingness to post more depth. On the other hand, a higher liquidity supply also triggers liquidity demand as the transaction costs of high volumes decline. Interestingly, this link does not necessarily hold for the relationship between depth and the number of trades which is negative in most cases. Hence, a high liquidity supply particularly triggers higher trade sizes but not necessarily a higher speed of trading. Likewise, a higher trading intensity tends to reduce first level depth. The estimates of $\hat{\mathbf{A}}_{2}$ and $\hat{\mathbf{A}}_{3}$ are negative reflecting some reversal effects in subsequent periods. As reflected by $\hat{\mathbf{B}}$, the processes are very persistent which is typical for high-frequency trading variables, see, e.g., Hautsch (2012). The Ljung-Box statistics 
of the resulting MEM residuals $\hat{\varepsilon}_{t}$ shown in Table 2 indicate that the specification captures the dynamics quite well. We observe that the Ljung-Box statistics are strongly reduced compared to the corresponding statistics for the raw data shown in Table 1 .

However, while dependencies in first moments seem to be successfully captured by the VMEM specification, dependencies in higher order moments are still present. This is reflected by quite high Ljung-Box statistics based on the residuals $\hat{\varepsilon}_{t}^{*}$ resulting from the Gaussian copula transformation (6). We observe that the mapping of MEM residuals into a Gaussian domain reveals serial dependencies which are not captured by a (linear) VMEM specification for plain variables. The presence of autocorrelations in transformed residuals provide hints on the existence of non-linearities in serial dependencies in trading characteristics. As the Gaussian copula transformation induces a mapping from $\mathbb{R}^{+}$to $\mathbb{R}$ and thus particularly affects small realizations of $\hat{\varepsilon}_{t}^{*}$, the autocorrelations in these residuals are presumably driven by distinct dependencies in unexpectedly small values of trading variables. These differences in dynamics of small and large realizations of volatility and liquidity variables might be captured by a - presumably complicate - non-linear VMEM specification for the trading variables directly. Nevertheless, even a highly flexible non-linear VMEM specification is probably unable to explain the strong dependencies in second moments of $\hat{\varepsilon}_{t}^{*}$ as revealed by the corresponding Ljung-Box statistics.

Table 4 shows the estimates of the VAR specification (8) based on $\hat{\varepsilon}_{t}^{*}$. Note that the reported standard errors do not explicitly account for the estimation errors in the previous stages. We justify this proceeding by the high number of observations yielding quite precise estimates of VMEM residuals and thus $F_{j} .{ }^{2}$ As suggested by the BIC and the Ljung-Box statistics of the resulting residuals $\boldsymbol{\eta}_{t}$ (Table 2), three lags are sufficient to capture the autocorrelations in $\hat{\varepsilon}_{t}^{*}$. We do not observe a particular dominance of dependencies on own histories but find that dependencies across the different trading characteristics are equally important. Hence, the copula transformation reveals evidence also for non-linear dependencies between the individual variables. However, the multivariate Ljung-Box statistics reported in Table 2 show that the VAR models does successfully remove both autocorrelations and cross-autocorrelations in the series.

For some series (particularly trading intensities) the Ljung-Box statistics of squared residuals $\hat{\varepsilon}_{t}^{* 2}$ and $\hat{\varepsilon}_{t}^{2}$ indicate even stronger dependencies in second moments than in first moments. As discussed above, the latter are associated with conditional variances of (normalized) trading variables and reflect the time-varying volatility of volatility and liquidity. These dynamics are

\footnotetext{
${ }^{2}$ Explicitly accounting for pre-stage estimation errors is analytically challenging but could be addressed numerically using bootstraping techniques.
} 
captured by the GARCH specification (10). The corresponding estimates based on fully specified matrices $\mathbf{A}_{1}^{h}$ and $\mathbf{B}_{1}^{h}$ of the lag order one are shown in Table 5 . We find significant presence of $(\mathrm{G}) \mathrm{ARCH}$ effects. This is most strongly seen if only diagonal specifications are estimated ${ }^{3}$. In the multivariate specification, however, these effects are overlaid by multi-collinearity effects reducing the significance of the innovation parameter estimates. Nevertheless, as also revealed by the estimates of $\mathbf{B}_{1}^{h}$, we find significant evidence for positive volatility spill-overs between all processes. Hence, the volatilities of volatility, liquidity demand and liquidity supply are crosscorrelated and moderately persistent over time. I.e., volatility shocks in liquidity demand - as reflected by an unexpectedly high or low trading intensity or market depth - spill over to other trading variables and increase uncertainty in liquidity supply and volatility and vice versa. These are states of the market where trading risks are high and trading costs are hard to predict. The reduction in Ljung-Box statistics of $\nu_{t}^{2}$ compared to those of $\hat{\varepsilon}_{t}^{2 *}$ (see Table 2) reflect that the (M)GARCH specification is able to capture these dependencies.

Nevertheless, the fact that the Ljung-Box statistics based on $\boldsymbol{\nu}_{t}^{2}$ are significant, reflects that dynamics in second moments are still not completely taken into account asking for a presumably even more flexible specification. Finally, as shown by the estimates of the DCC parameters, we find significant evidence for dynamics in conditional correlations between (transformed) trading variables. Estimates of $\hat{\delta}$ being close to one indicate a high persistence in correlation processes - very similar to that of daily asset return correlations. The residuals stemming from the DCC process are computed based on the Cholesky transformation $\boldsymbol{\nu}_{t}^{*}=\mathbf{R}_{t}^{-1 / 2} \boldsymbol{\nu}_{t}$. Not surprisingly, the Ljung-Box statistics of $\boldsymbol{\nu}_{t}^{*}$ are very similar to those of $\boldsymbol{\nu}_{t}$ showing that dependencies in first moments are removed while slight dependencies in second moments are still present. Nevertheless, it turns out that the DCC specification is successful in removing the mutual correlations between the series. This is revealed by applying John's test for zero mutual correlation based on the empirical distance between the sample covariance matrix and a diagonal matrix (John (1971)). The corresponding test statistic is given by

$$
T_{J}=\frac{1}{K} \operatorname{tr}\left[\left(\frac{\mathbf{S}}{(1 / K) \operatorname{tr}(\mathbf{S})}-\mathbf{I}\right)^{2}\right],
$$

where $\mathbf{S}$ is the sample covariance matrix based on the residual series. As shown by Ledoit and Wolf (2002), the asymptotic ( $\left.\chi^{2}\right)$ distribution of the test statistic is also valid if the cross-sectional dimension $K$ relative to the sample size $T$ becomes high. Table 2 reports that the test statistics are strongly reduced by the DCC specification.

\footnotetext{
${ }^{3}$ The results are not shown here.
} 
To check the adequacy of the Gaussian copula, we test the normality of the residual series. Figure 1 depicts the the kernel density estimates (using an Epanechnikov kernel with optimal bandwidth) for all residual series $\left\{\varepsilon_{t}\right\},\left\{\varepsilon_{t}^{*}\right\},\left\{\boldsymbol{\eta}_{t}\right\},\left\{\boldsymbol{\nu}_{t}\right\},\left\{\boldsymbol{\nu}_{t}^{*}\right\}$ for the number of trades in AIG trading. We compare the kernel density estimates with the density of a normal distribution fitted to the corresponding series. Not surprisingly, for $\left\{\varepsilon_{t}\right\}$, the kernel density estimates significantly depart from the correspondingly fitted normal distribution. Conversely, the distribution of $\left\{\varepsilon_{t}^{*}\right\}$ is visually hardly distinguishable from Gaussianity. The results are representative for the other residual series as well as for all other stocks.

To formally test for normality, we apply a Kolmogorov-Smirnov test based on $\boldsymbol{\nu}_{t}^{*}$. As shown by Table 6, normality cannot be rejected for nearly all series. The only exception is observed for the residual series stemming from trade counts where the assumption of normality is rejected in four out of five cases. Nevertheless, overall empirical evidence strongly supports the normality of transformed residuals and thus backs the usefulness of a Gaussian copula. To test for the joint normality of the individual series, we test the distribution of the statistic

$$
\xi_{t}=\nu_{1, t}^{* 2}+\nu_{2, t}^{* 2}+\nu_{3, t}^{* 2}+\nu_{4, t}^{* 2}
$$

against a $\chi^{2}$-distribution with 4 degrees of freedom. Corresponding quantile-quantile (QQ)plots along with associated p-values of Kolmogorov-Smirnov statistics are depicted by Figure 2 The plots reveal close correspondance of theoretical and empirical quantiles for major parts of the distributions. However, deviations are depicted for extreme observations in the right tails. Consequently, joint normality of all series in form of a $\chi_{4}^{2}$ distribution for $\xi_{t}$ cannot be rejected on a $1 \%$ level only. This is presumably driven by deviations from Gaussianity for the series of trade counts and very few extreme observations whose distributional properties are hard to capture. Finally, Table 7 gives the values of the likelihood function and the Bayes Information Criteria (BIC) for the basic and extended VMEM specifications. It turns out that the model's goodnessof-fit is clearly improved when dynamics in higher order moments are taken into account.

Figure 3 plots the estimated conditional variances $\mathbf{h}_{t}$ and conditional correlations $\mathbf{R}_{t}$ of $\boldsymbol{\eta}_{t}$ for the stock AIG. We observe significant short-term and long-term movements of conditional variances over time. Induced by the positive spill-overs, we observe that the series tend to move in lock-steps. This is particularly true for the volatilities of midquote volatility and of cumulative trade sizes. As depicted by Figure 4 , the conditional correlations between the individual series reveal significant time variations as well. Not surprisingly, the highest (positive) correlations are shown between cumulative volumes and and trade counts which are correlated by construction. 
Likewise, positive mutual dependencies are identified between midquote volatility and cumulative volumes as well as trade counts. Confirming the VMEM estimates above, dependencies between first level depth and volatility as well as trade counts tend to be negative or close to zero, respectively. Hence, liquidity supply is reduced when volatility and the number of trades are high. Conversely, liquidity supply is positively autocorrelated with cumulative trading volumes confirming the positive dependencies between liquidity demand and supply.

\section{Conclusions}

In this paper, we propose extending a VMEM model to capture dynamics in conditional (co-) variances of multivariate trading processes. The key idea is to transform innovations stemming from a VMEM specification using a Gaussian copula. The mapping into a Gaussian domain allows to naturally disentagle dynamics in first and second conditional moments. We propose modeling the latter using a DCC-GARCH process which is tractable also in high dimensions. The model is estimated using a composite maximum likelihood approach and is easily extended in various directions.

Applying the new approach to model 5 min volatility, cumulative trading volumes, trade counts as well as first level depth based on various stocks traded at the NYSE, we show the following results. Firstly, the proposed Gaussian copula is empirically supported by the data as the normality of transformed VMEM innovations cannot be rejected in most cases. Secondly, we find significant evidence for non-linear (cross-)dependencies between trading variables. Third, trading variables reveal time-varying conditional variances reflecting the riskiness in liquidity

supply, demand and volatility. The processes are persistent and are subject to positive crossdependencies. I.e., uncertainty in trading characteristics easily spills over between the individual components. Fourth, we find time-varying and persistent conditional correlations between the processes. Fifth, according to information criteria and residual diagnostics, the copula-based multiplicative error process yields a better goodness-of-fit and a better description of higherorder dynamics in multivariate trading processes.

\section{References}

Bauwens, L., S. Laurent, and J. Rombouts (2006): "Multivariate GARCH models: a survey," Journal of Applied Econometrics, 21, 79-109. 
Bauwens, L., And D. Veredas (2004): "The Stochastic Conditional Duration Model: A Latent Factor Model for the Analysis of Financial Durations," Journal of Econometrics, 119, $381-412$.

Bollerslev, T. (1986): "Generalized Autoregressive Conditional Heteroskedasticity," Journal of Econometrics, 31, 307-327.

Brownlees, C., F. Cipollini, and G. M. Gallo (2010): "Intra-daily Volume Modeling and Prediction for Algorithmic Trading," Journal of Financial Econometrics, forthcoming.

Brownlees, C., And M. VAnnucci (2010): “A Bayesian Approach for Capturing Daily Heterogeneity in Intra-Daily Durations Time Series: The Mixed Autoregressive Conditional Duration Model,” Working Paper, Stern School of Business, New York University.

Cipollini, F., R. F. Engle, and G. M. Gallo (2007): "Vector Multiplicative Error Models: Representation and Inference,” Working Paper, University of Florence.

Engle, R., And K. Sheppard (2001): “Theoretical and Empirical Properties of Dynamic Conditional Correlation Multivariate GARCH,” NBER Working Paper 8554.

Engle, R. F. (1982): "Autoregressive Conditional Heteroscedasticity with Estimates of the Variance of United Kingdom Inflation,” Econometrica, 50, 987-1006.

ENGLE, R. F. (2000): “The Econometrics of Ultra-High-Frequency Data,” Econometrica, 68, 1, $1-22$.

Engle, R. F. (2002a): “Dynamic Conditional Correlation,” Journal of Business and Economic Statistics, 20, 339-350.

Engle, R. F. (2002b): "New Frontiers for ARCH Models," Journal of Applied Econometrics, $17,425-446$.

Engle, R. F., And J. R. Russell (1998): “Autoregressive Conditional Duration: A New Model for Irregularly Spaced Transaction Data," Econometrica, 66, 1127-1162.

Ghysels, E., C. GouriérouX, And J. JASiAK (1998): “Stochastic Volatility Duration Models," Discussion paper, CIRANO.

(2004): “Stochastic Volatility Duration Models," Journal of Econometrics, 119, 413433. 
Glosten, L. R. (1994): “Is the Electronic Open Limit Order Book Inevitable," The Journal of Finance, 49, 1127-1161.

Hautsch, N. (2008): "Capturing Common Components in High-Frequency Financial Time Series: A Multivariate Stochastic Multiplicative Error Model," Journal of Economic Dynamics \& Control, 32, 3978-4009.

- (2012): Econometrics of Financial High-Frequency Data, forthcoming. Springer, Berlin.

JASIAK, J. (1998): “Persistence in Intratrade Durations," Finance, 19, 166-195.

JoE, H. (1997): Multivariate Models and Dependence Concepts. Chapman \& Hall, London.

- (2005): "Asymptotic efficiency of the two-stage estimation method for copula-based models," Journal of Multivariate Analysis, 94, 401-419.

JoHn, S. (1971): “Some optimal multivariate tests," Biometrika, 58, 123-127.

Jondeau, E., And M. Rockinger (2006): "The Copula-GARCH model of conditional dependencies: an international stock market application," Journal of International Money and Finance, 25, 827-853.

Karanasos, M. (2004): “The Statistical Properties of Long-memory ACD Models,” WSEAS Transactions on Business and Economics, 2, 169-175.

LEDOIT, O., AND M. Wolf (2002): "Some hypothesis tests for the covariance matrix when the dimension is large compared to the sample size," The Annals of Statistics, 30, 1081-1102.

LEE, T.-H., AND X. LONG (2009): "Copula-based multivariate GARCH model with uncorrelated dependent errors," Journal of Econometrics, 150, 207-218.

LindSAY, B. (1988): “Composite likelihood methods,” Contemporary Mathematics, 80, 220 239.

LIU, Y., AND R. LUGER (2009): "Efficient estimation of copula-GARCH models," Computational Statistics \& Data Analysis, 53, 2284-2297.

Manganelli, S. (2005): "Duration, Volume and Volatility Impact of Trades," Journal of Financial Markets, 8, 377-399. 
Meitz, M., AND T. TERÄSvirTA (2006): "Evaluating Models of Autoregressive Conditional Duration," Journal of Business \& Economic Statistics, 24, 104-124.

NG, C., H. Joe, D. Karlis, And J. LiU (2011): “Composite likelihood for time series models with a latent autoregressive process," Statistica Sinica, 21, 279-305.

Patton, A. (2001): "Modelling Time-Varying Exchange Rate Dependence using the Conditional Copula," University of California Discussion Paper 2001-09.

(2006): "Modelling asymmetric exchange rate dependence," International Economic Review, 47, 527-556.

TAYLOR, S. J. (1982): "Financial Returns Modelled by the Product of Two Stochastic Processes - A Study of Daily Sugar Prices," in Time Series Analysis: Theory and Practice, ed. by O. D. Anderson. North-Holland, Amsterdam.

White, H. (1996): Estimation, Inference, and Specification Analysis. Cambridge University Press, Cambridge.

ZhANG, M. Y., J. RusselL, AND R. S. TSAY (2001): “A Nonlinear Autoregressive Conditional Duration Model with Applications to Financial Transaction Data," Journal of Econometrics, 104, 179-207. 


\section{Appendix}

\begin{tabular}{lrrrr}
\hline \hline \multicolumn{5}{c}{ AIG } \\
\hline Mean & Returns & Volumes & Trades & Depth \\
S.D. & 17.412 & 54.960 & 43.131 & 22.086 \\
$1 \%$ & -27.383 & 14.700 & 23.000 & 6.567 \\
5\% & 27.660 & 135.615 & 67.000 & 57.322 \\
LB(20) & 2628 & 41369 & 44629 & 50653 \\
& \multicolumn{4}{c}{ AMR } \\
\hline Mean & -0.032 & 46.486 & 34.075 & 21.703 \\
S.D. & 49.987 & 40.672 & 13.064 & 37.150 \\
1\% & -79.889 & 9.100 & 15.000 & 6.978 \\
5\% & 80.067 & 121.400 & 58.000 & 48.625 \\
LB(20) & 2055 & 37089 & 50179 & 16873 \\
& & ASH & & \\
\hline Mean & 0.127 & 9.149 & 20.861 & 5.784 \\
S.D. & 25.164 & 8.651 & 9.898 & 4.944 \\
1\% & -38.981 & 1.800 & 7.000 & 2.232 \\
5\% & 39.802 & 23.500 & 39.000 & 13.697 \\
LB(20) & 4630 & 36178 & 39364 & 15266 \\
& \multicolumn{5}{c}{ Boeing } & & \\
\hline Mean & -0.036 & 30.795 & 38.438 & 10.041 \\
S.D. & 23.138 & 24.495 & 13.472 & 9.527 \\
1\% & -36.538 & 7.800 & 19.000 & 3.915 \\
5\% & 36.355 & 76.700 & 63.000 & 23.056 \\
LB(20) & 2583 & 40728 & 52566 & 6943 \\
& & Citigroup & & \\
\hline Mean & -0.050 & 103.724 & 45.847 & 53.410 \\
S.D. & 17.390 & 88.271 & 13.479 & 56.242 \\
1\% & -27.546 & 28.200 & 26.000 & 16.040 \\
5\% & 27.960 & 249.900 & 69.000 & 129.695 \\
LB(20) & 3028 & 36924 & 60612 & 30895 \\
\hline \hline & & & & \\
\hline
\end{tabular}

Table 1: Summary statistics of annualized 5min returns, cumulative trading volumes (in 1000), number of trades and average first level depth of the stocks AIG, Citigroup, Boeing, ASH and AMR. The table shows means, standard deviations and 5\% and 95\% quantiles. Moreover, LjungBox statistics based on 20 lags of the corresponding de-seasonalized series are shown. In case of returns, the Ljung-Box statistics of squared $\operatorname{ARMA}(1,1)$ residuals are displayed. 


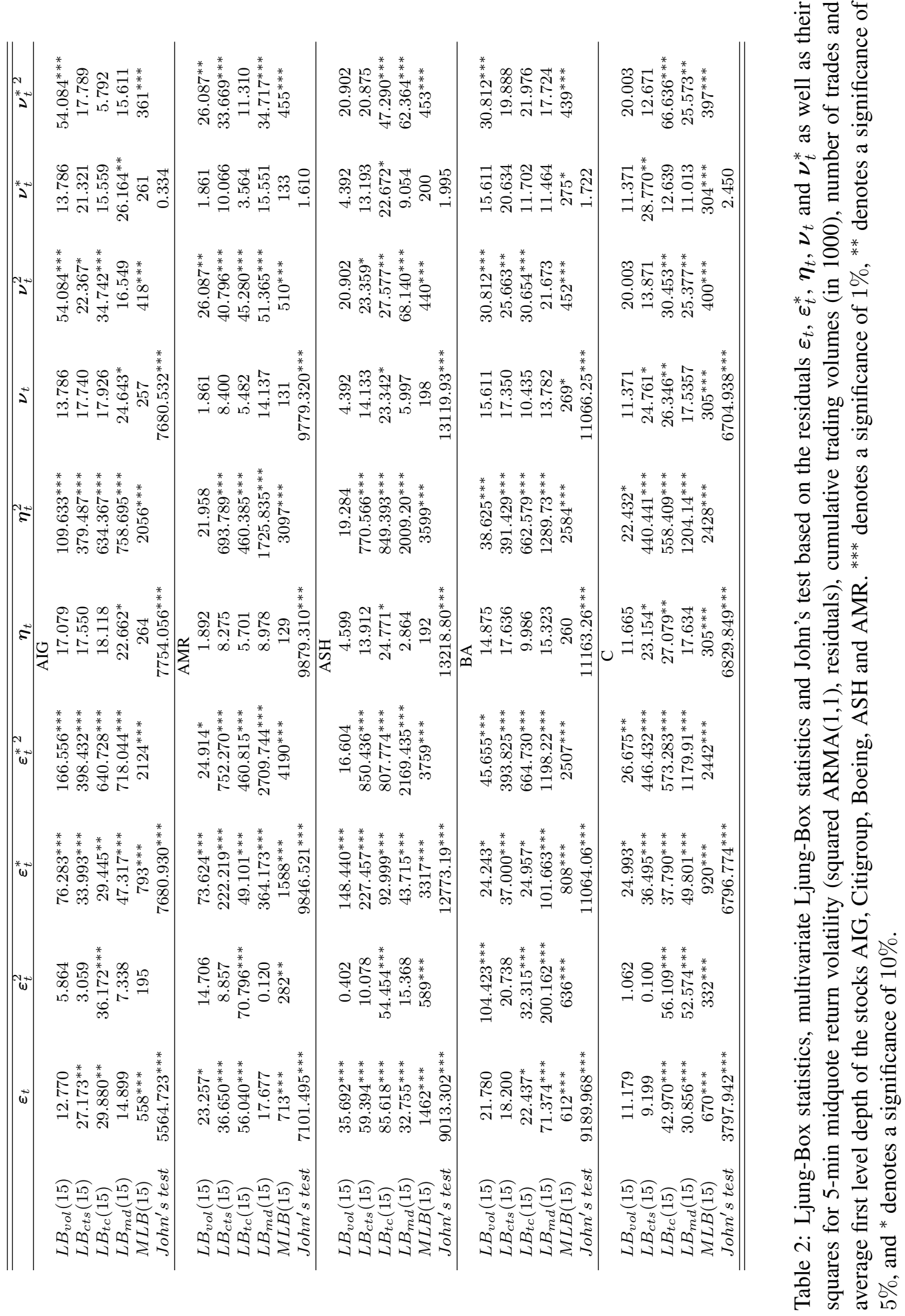




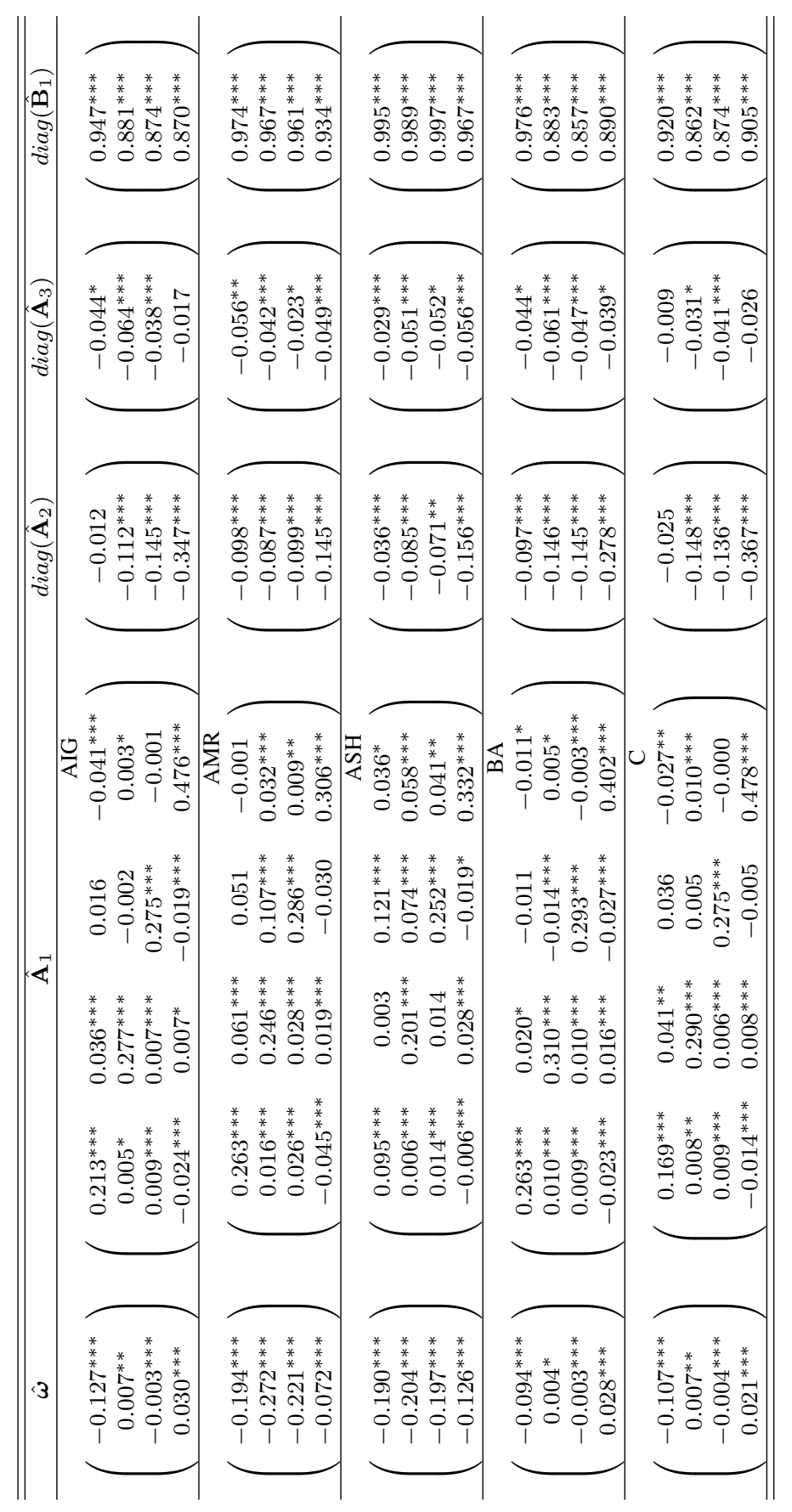

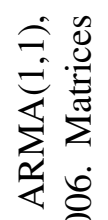

己

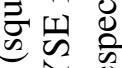
之文:

豞 考

E

记

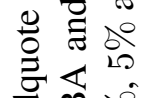

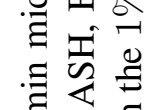

ค่

可交

불

ก 《 कू

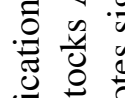

ป ญ व

की

$\sum_{i=1} \underbrace{0}$

$\sum^{\prime} \frac{\overline{2}}{0} *$

$\stackrel{0}{ \pm} 0^{*}$

$\Xi$ ত্তু

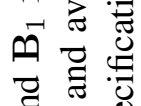

उี ज्ञ

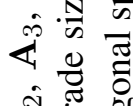

«ं

<艺导

$3 \stackrel{0}{7}$

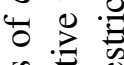

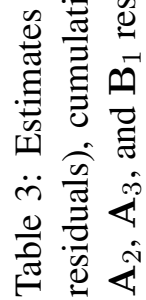




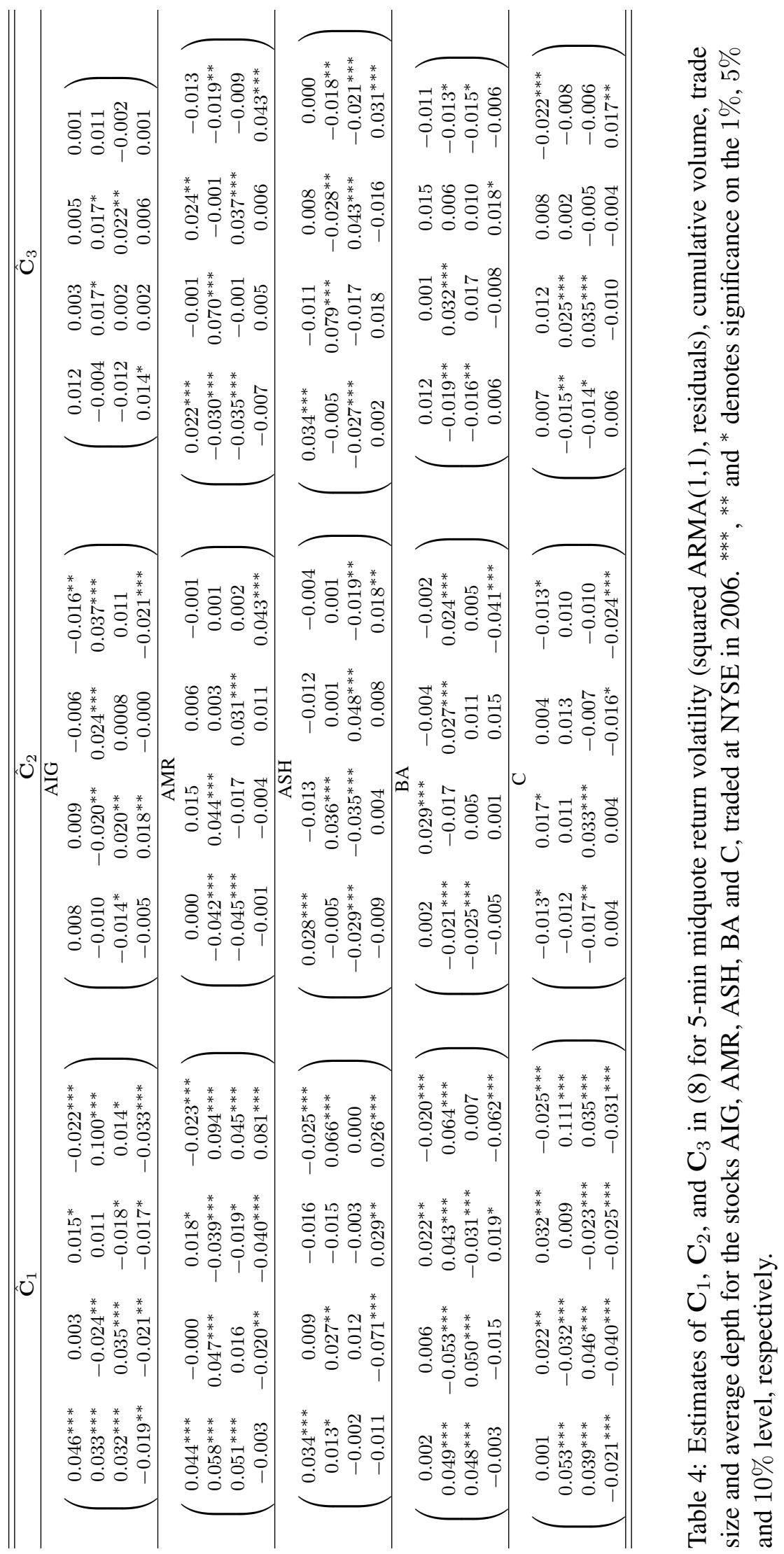




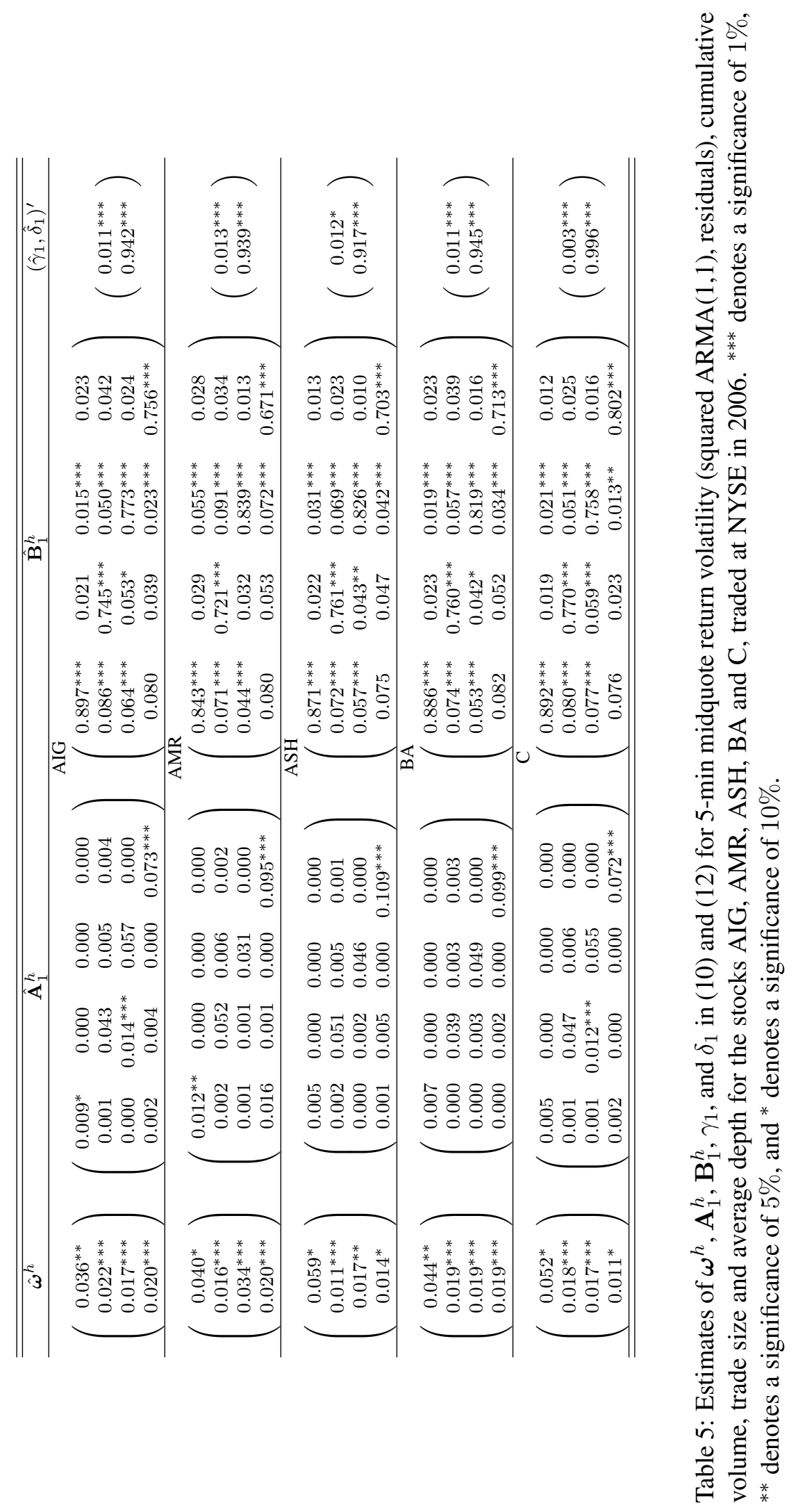




\begin{tabular}{cccc}
\hline \hline $\boldsymbol{\nu}_{1, t}^{*}$ & $\boldsymbol{\nu}_{2, t}^{*}$ & $\boldsymbol{\nu}_{3, t}^{*}$ & $\boldsymbol{\nu}_{4, t}^{*}$ \\
\hline \multicolumn{4}{c}{ AIG } \\
0.672 & $0.996 \quad 0.029$ & 0.696 \\
\multicolumn{4}{c}{ AMR } \\
0.528 & $0.789 \quad 0.001$ & 0.527 \\
\multicolumn{4}{c}{ ASH } \\
0.932 & $0.198 \quad 0.000$ & 0.031 \\
\multicolumn{4}{c}{ BA } \\
1 & $0.437 \quad 0.000$ & 0.356 \\
0.977 & 0.829 & 0.060 & 0.478 \\
\hline \hline
\end{tabular}

Table 6: $p$-values of the Kolmogorov-Smirnov test statistic to test the null hypothesis of the univariate standard normal distribution for each component of the residual vector $\boldsymbol{\nu}_{t}^{*}=\mathbf{R}_{t}^{-1 / 2} \boldsymbol{\nu}_{t}$ for 5-min midquote return volatility (squared $\operatorname{ARMA}(1,1)$, residuals), cumulative volume, trade size and average depth for the stocks AIG, AMR, ASH, BA and C, traded at NYSE in 2006.

\begin{tabular}{ccc}
\hline \hline$L L H_{\text {ext }}$ & $L L H_{\text {basic }}$ BIC $C_{\text {ext }}$ & BIC basic \\
\hline \multicolumn{5}{c}{ AIG } \\
-61367.19 & $-66505.93 \quad 123891.7$ & 133325.7 \\
AMR \\
-59824.11 & $-66762.93 \quad 120805.5$ & 133839.7 \\
ASH \\
-55759.49 & $-66406.21 \quad 112676.3$ & 133126.3 \\
-60088.01 & $-67651.26 \quad 121333.3$ & 135616.4 \\
-62967.98 & $-67571.4 \quad 127093.3$ & 135456.7 \\
\hline \hline
\end{tabular}

Table 7: Likelihood values and BIC values for the basic and extended VMEM specifications for 5-min midquote return volatility (squared $\operatorname{ARMA}(1,1)$, residuals), cumulative volume, trade size and average depth for the stocks AIG, AMR, ASH, BA and C, traded at NYSE in 2006. 
Figure 1. Kernel density estimates (Epanechnikov kernel) for the residuals $\left\{\varepsilon_{t}\right\},\left\{\varepsilon_{t}^{*}\right\},\left\{\boldsymbol{\eta}_{t}\right\}$, $\left\{\boldsymbol{\nu}_{t}\right\}$, and $\left\{\boldsymbol{\nu}_{t}^{*}\right\}$ based on 5-min midquote return volatility (squared ARMA(1,1), residuals; 'vol'), cumulative volume ('cv'), trade size ('ts') and average depth ('ad') for AIG, traded at NYSE in 2006. The black solid line depicts the kernel density estimate while the grey dashed line represents the density of a normal distribution fitted to the corresponding series.
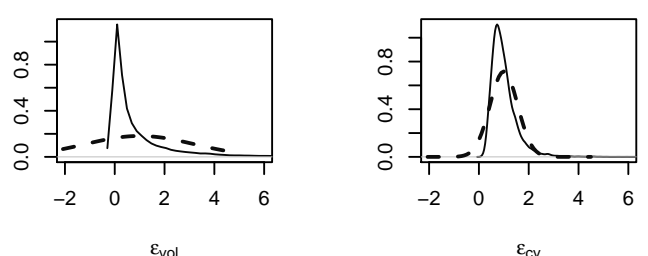

$\varepsilon_{\mathrm{cV}}$
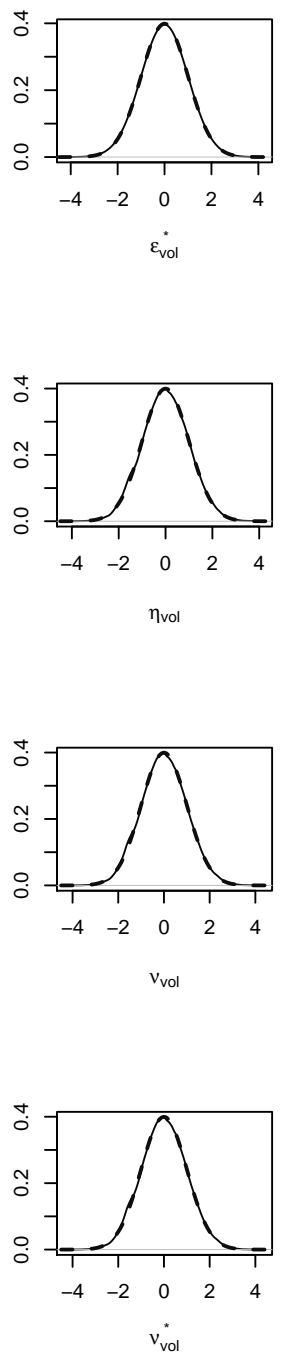

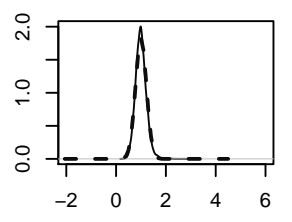

$\varepsilon_{\mathrm{ts}}$
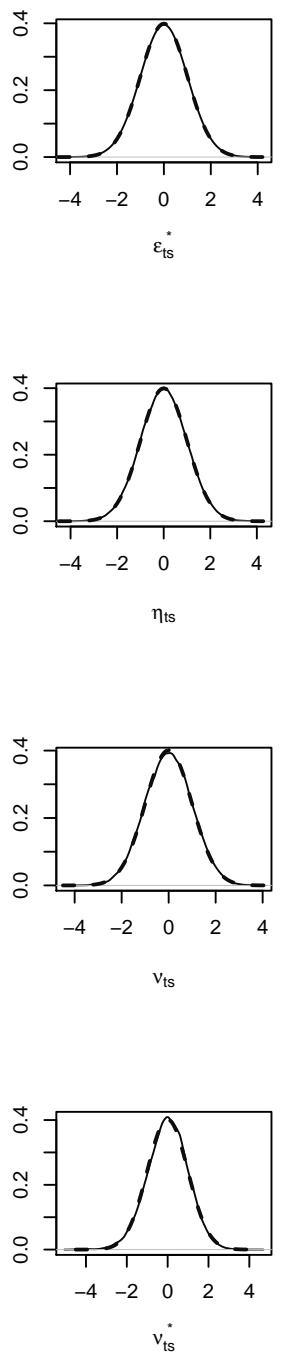

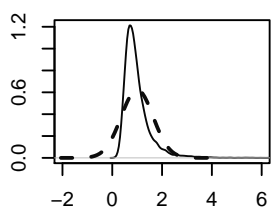

$\varepsilon_{\mathrm{ad}}$
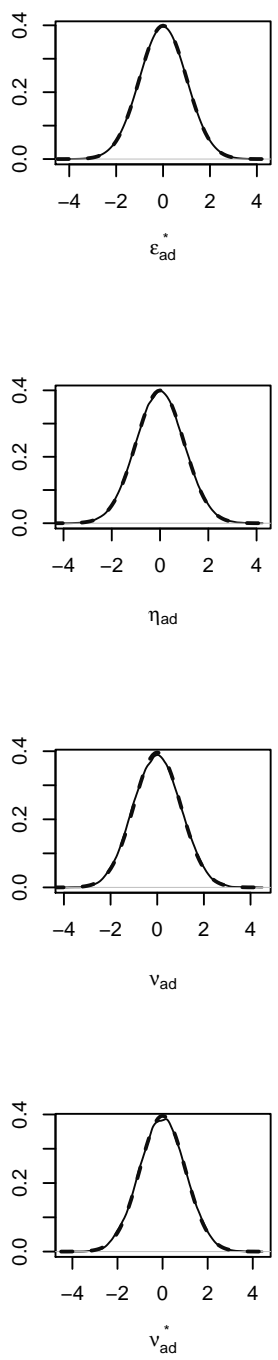
Figure 2. QQ-plots of $\xi_{t}=\nu_{1, t}^{* 2}+\nu_{2, t}^{* 2}+\nu_{3, t}^{* 2}+\nu_{4, t}^{* 2}$ versus the theoretical quantiles of the $\chi^{2}$ distribution with 4 degrees of freedom. The underlying residual series stem from 5-min midquote return volatility (squared ARMA(1,1), residuals), cumulative volume, trade size and average depth for the stocks AIG, AMR, ASH, BA and C, traded at NYSE in 2006. p-values of KolmogorovSmirnov test statistics for the null hypothesis a $\chi_{4}^{2}$-distribution are provided in the headers.
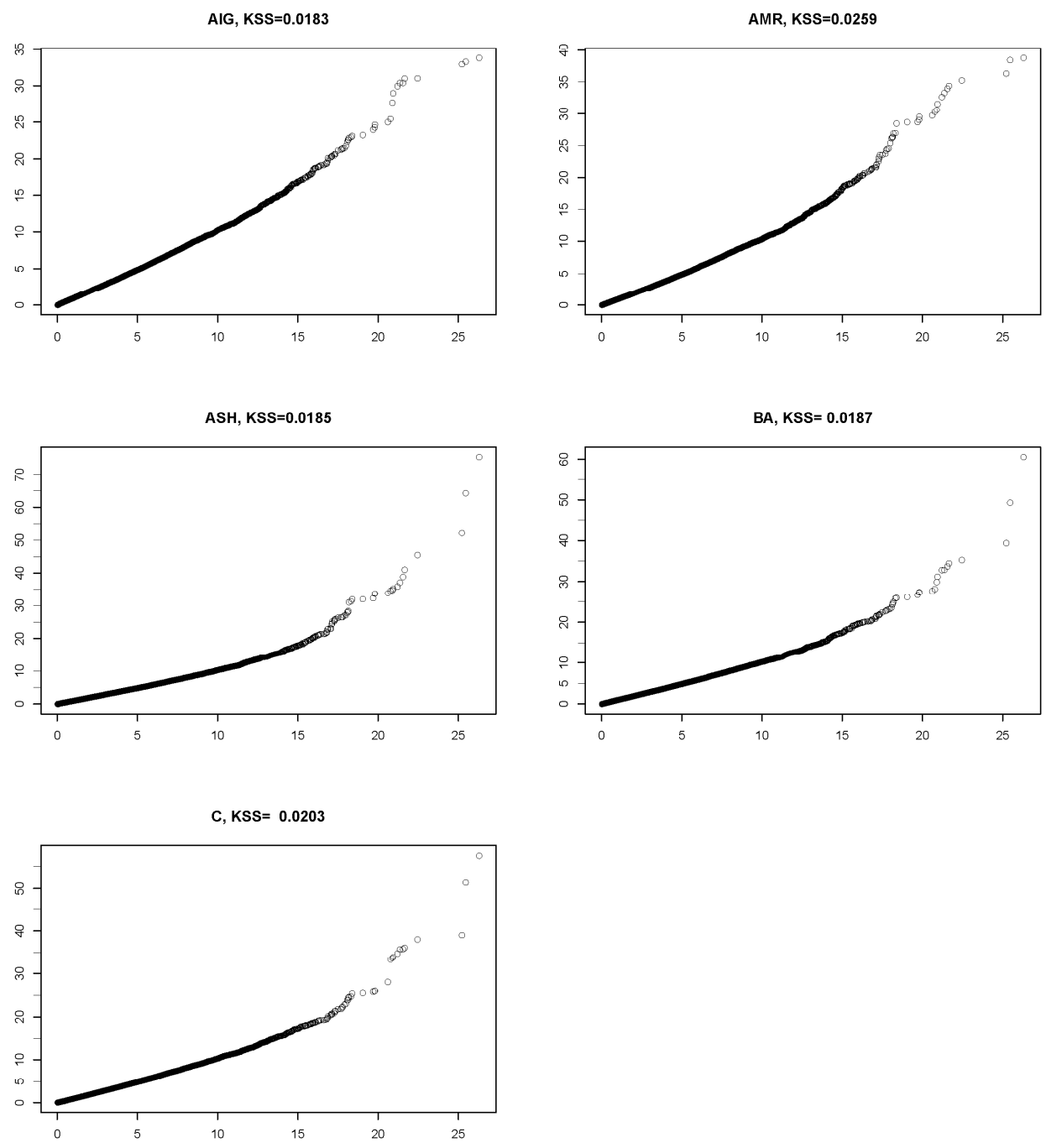
Figure 3. Estimated conditional variance processes $\left\{h_{., t}\right\}$ of 5-min midquote return volatility (top left), cumulative volumes (top right), the number of trades (bottom left) and the average first level depth (bottom right) for AIG, traded at NYSE, 2006.
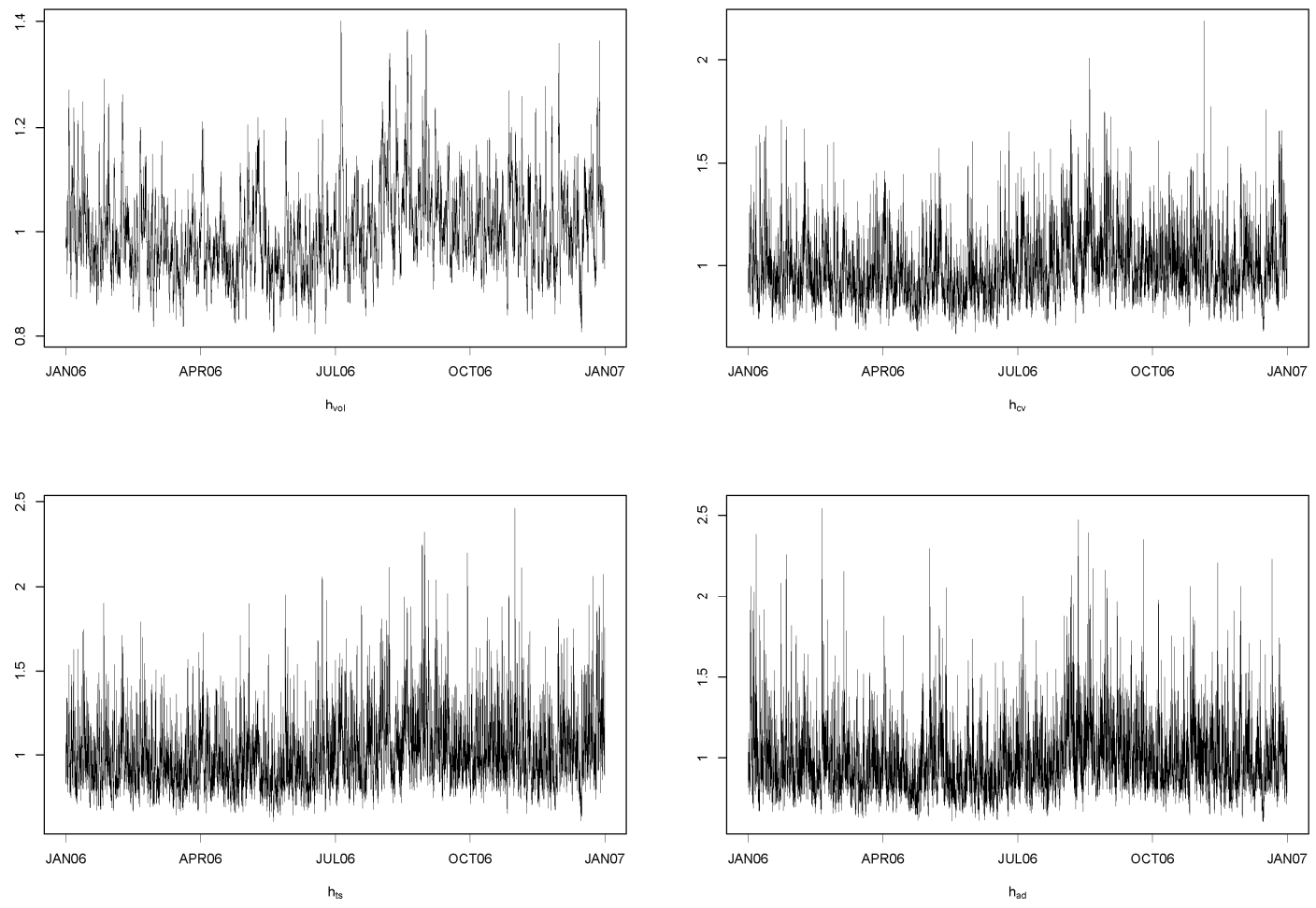
Figure 4. Conditional correlations $\left\{R_{., ; t}\right\}$ between 5-min midquote return volatility and cumulative trading volumes (top left), 5-min midquote return volatility and the number of trades (top right), 5-min midquote return volatility and average first level depth (middle left), cumulative volumes and number of trades (middle right), cumulative volumes and average first level depth (bottom left), the number of trades and average first level depth (bottom right) for AIG, traded at NYSE, 2006.
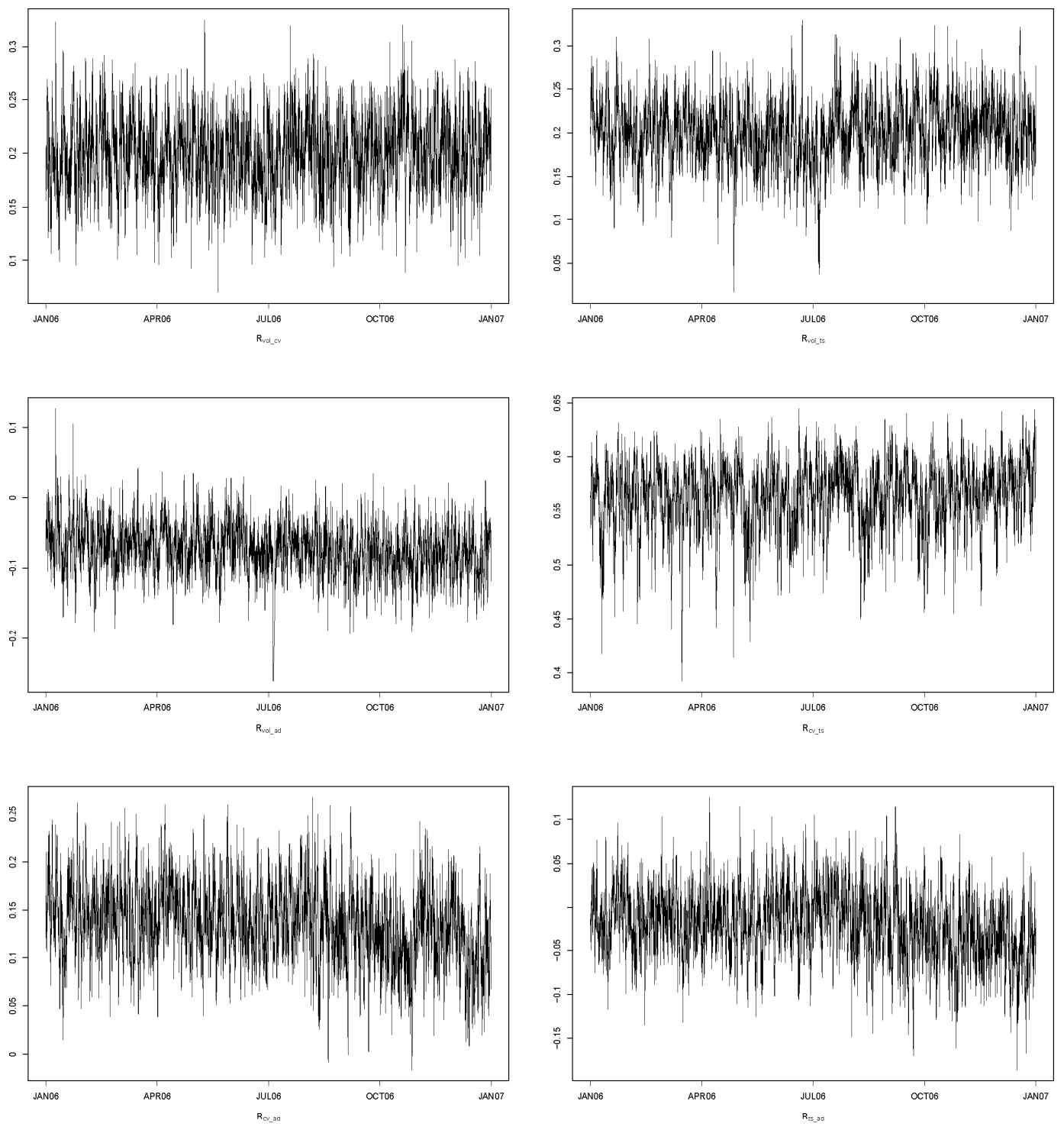


\section{SFB 649 Discussion Paper Series 2012}

For a complete list of Discussion Papers published by the SFB 649, please visit http://sfb649.wiwi.hu-berlin.de.

001 "HMM in dynamic HAC models" by Wolfgang Karl Härdle, Ostap Okhrin and Weining Wang, January 2012.

002 "Dynamic Activity Analysis Model Based Win-Win Development Forecasting Under the Environmental Regulation in China" by Shiyi Chen and Wolfgang Karl Härdle, January 2012.

003 "A Donsker Theorem for Lévy Measures" by Richard Nickl and Markus Reiß, January 2012.

004 "Computational Statistics (Journal)" by Wolfgang Karl Härdle, Yuichi Mori and Jürgen Symanzik, January 2012.

005 "Implementing quotas in university admissions: An experimental analysis" by Sebastian Braun, Nadja Dwenger, Dorothea Kübler and Alexander Westkamp, January 2012.

006 "Quantile Regression in Risk Calibration" by Shih-Kang Chao, Wolfgang Karl Härdle and Weining Wang, January 2012.

007 "Total Work and Gender: Facts and Possible Explanations" by Michael Burda, Daniel S. Hamermesh and Philippe Weil, February 2012.

008 "Does Basel II Pillar 3 Risk Exposure Data help to Identify Risky Banks?" by Ralf Sabiwalsky, February 2012.

009 "Comparability Effects of Mandatory IFRS Adoption" by Stefano Cascino and Joachim Gassen, February 2012.

010 "Fair Value Reclassifications of Financial Assets during the Financial Crisis" by Jannis Bischof, Ulf Brüggemann and Holger Daske, February 2012.

011 "Intended and unintended consequences of mandatory IFRS adoption: A review of extant evidence and suggestions for future research" by Ulf Brüggemann, Jörg-Markus Hitz and Thorsten Sellhorn, February 2012.

012 "Confidence sets in nonparametric calibration of exponential Lévy models" by Jakob Söhl, February 2012.

013 "The Polarization of Employment in German Local Labor Markets" by Charlotte Senftleben and Hanna Wielandt, February 2012.

014 "On the Dark Side of the Market: Identifying and Analyzing Hidden Order Placements" by Nikolaus Hautsch and Ruihong Huang, February 2012.

015 "Existence and Uniqueness of Perturbation Solutions to DSGE Models" by Hong Lan and Alexander Meyer-Gohde, February 2012.

016 "Nonparametric adaptive estimation of linear functionals for low frequency observed Lévy processes" by Johanna Kappus, February 2012.

017 "Option calibration of exponential Lévy models: Implementation and empirical results" by Jakob Söhl und Mathias Trabs, February 2012.

018 "Managerial Overconfidence and Corporate Risk Management" by Tim R. Adam, Chitru S. Fernando and Evgenia Golubeva, February 2012.

019 "Why Do Firms Engage in Selective Hedging?" by Tim R. Adam, Chitru S. Fernando and Jesus M. Salas, February 2012.

020 "A Slab in the Face: Building Quality and Neighborhood Effects" by Rainer Schulz and Martin Wersing, February 2012.

021 "A Strategy Perspective on the Performance Relevance of the CFO" by Andreas Venus and Andreas Engelen, February 2012.

022 "Assessing the Anchoring of Inflation Expectations" by Till Strohsal and Lars Winkelmann, February 2012.

\section{SFB 649, Spandauer Straße 1, D-10178 Berlin http: //sfb649.wiwi.hu-berlin.de}

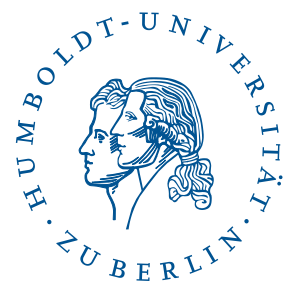




\section{SFB 649 Discussion Paper Series 2012}

For a complete list of Discussion Papers published by the SFB 649, please visit http://sfb649.wiwi.hu-berlin.de.

023 "Hidden Liquidity: Determinants and Impact" by Gökhan Cebiroglu and Ulrich Horst, March 2012.

024 "Bye Bye, G.I. - The Impact of the U.S. Military Drawdown on Local German Labor Markets" by Jan Peter aus dem Moore and Alexandra Spitz-Oener, March 2012.

025 "Is socially responsible investing just screening? Evidence from mutual funds" by Markus Hirschberger, Ralph E. Steuer, Sebastian Utz and Maximilian Wimmer, March 2012.

026 "Explaining regional unemployment differences in Germany: a spatial panel data analysis" by Franziska Lottmann, March 2012.

027 "Forecast based Pricing of Weather Derivatives" by Wolfgang Karl Härdle, Brenda López-Cabrera and Matthias Ritter, March 2012.

028 "Does umbrella branding really work? Investigating cross-category brand loyalty" by Nadja Silberhorn and Lutz Hildebrandt, April 2012.

029 "Statistical Modelling of Temperature Risk" by Zografia Anastasiadou, and Brenda López-Cabrera, April 2012.

030 "Support Vector Machines with Evolutionary Feature Selection for Default Prediction" by Wolfgang Karl Härdle, Dedy Dwi Prastyo and Christian Hafner, April 2012.

031 "Local Adaptive Multiplicative Error Models for High-Frequency Forecasts" by Wolfgang Karl Härdle, Nikolaus Hautsch and Andrija Mihoci, April 2012.

032 "Copula Dynamics in CDOs." by Barbara Choroś-Tomczyk, Wolfgang Karl Härdle and Ludger Overbeck, May 2012.

033 "Simultaneous Statistical Inference in Dynamic Factor Models" by Thorsten Dickhaus, May 2012.

034 "Realized Copula" by Matthias R. Fengler and Ostap Okhrin, Mai 2012.

035 "Correlated Trades and Herd Behavior in the Stock Market" by Simon Jurkatis, Stephanie Kremer and Dieter Nautz, May 2012

036 "Hierarchical Archimedean Copulae: The HAC Package" by Ostap Okhrin and Alexander Ristig, May 2012.

037 "Do Japanese Stock Prices Reflect Macro Fundamentals?" by Wenjuan Chen and Anton Velinov, May 2012.

038 "The Aging Investor: Insights from Neuroeconomics" by Peter N. C. Mohr and Hauke R. Heekeren, May 2012.

039 "Volatility of price indices for heterogeneous goods" by Fabian Y.R.P. Bocart and Christian M. Hafner, May 2012.

040 "Location, location, location: Extracting location value from house prices" by Jens Kolbe, Rainer Schulz, Martin Wersing and Axel Werwatz, May 2012.

041 "Multiple point hypothesis test problems and effective numbers of tests" by Thorsten Dickhaus and Jens Stange, June 2012

042 "Generated Covariates in Nonparametric Estimation: A Short Review." by Enno Mammen, Christoph Rothe, and Melanie Schienle, June 2012.

043 "The Signal of Volatility" by Till Strohsal and Enzo Weber, June 2012.

044 "Copula-Based Dynamic Conditional Correlation Multiplicative Error Processes" by Taras Bodnar and Nikolaus Hautsch, July 2012

\section{SFB 649, Spandauer Straße 1, D-10178 Berlin http://sfb649.wiwi.hu-berlin.de}

